\section{SAT0105 PRELIMINARY ANALYSIS OF GENETIC VARIANTS IN THE IMMUNE SYSTEM RELATED TO THE BODY MASS INDEX IN EARLY ARTHRITIS PATIENTS}

P. Moreno ${ }^{1}$, N. Montes ${ }^{1}$, J. Martín ${ }^{2}$, D. Carmona ${ }^{3}$, C. M.Mora ${ }^{4}$, R. P.Gomáriz ${ }^{5}$, A. Lamana ${ }^{1}$, A. Triguero ${ }^{1}$, A. M. Ortiz ${ }^{1}$, I. G. Álvaro ${ }^{11}$ Rheumatology, HU La Princesa, Madrid; ${ }^{2}$ Instituto López Neyra - CSIC; ${ }^{3}$ Genetics Department, Universidad Granada, Granada; ${ }^{4}$ Facultad de Medicina - UCM; ${ }^{5}$ Facultad de Biología - UCM, Madrid, Spain

Background: We have observed in previous analyzes in our early arthritis (EA) cohort that patients with a higher body mass index (BMI) are, more frequently, ACPA negative and these patients carry, with a lower frequency, HLADRB1 alleles that encode for the shared epitope.

Objectives: To identify SNPs (Single Nucleotide Polymorphisms) of immune system genes related to $\mathrm{BMI}$ in EA patients.

Methods: The 257 patients of the PEARL (Princess Early Arthritis Register Longitudinal) cohort in which high density genotyping was available (using the Immunochip array of llumina Inc) were included. As a previous step, those SNPs that did not meet the requirements of a genotyping call rate lower than $98 \%$, being out of Hardy-Weinberg equilibrium $\left(p<10-{ }^{4}\right)$ and minor allele frequency lower than $1 \%$ were excluded. IMPUTE v.2 was used for the genotype imputation of the SNPS that failed in the immunochip, using as reference the data of phase III of the 1000 $G$ project. The association analysis of the remaining SNPs was made by linear regression adjusted by sex, age and study level with PLINK v1.9. Of the 1384 SNPs associated with BMI with a value of $p<0.01,250$ SNPs were selected according to the lowest values of the division of $p$ divided by the absolute value of its $\beta$ coefficient. After analyzing and excluding the SNPS that were in linkage desequilibrium, the importance of the 186 resulting SNPs was quantified with the "Random Forest" and "Boosted Regression Tree" techniques using \%IncMSE (Mean Decrease Accuracy).

Results: Table 1 shows the selection of the 15 SNPs that were more important in both "machine learning" techniques according to BMI. Although most of these SNPs are located in non-coding regions (intergenic or intronic), some of the genes where the SNPs belong or the neighboring genes have shown association in some GWAS (Genome-Wide Association) with a minor (BMP7) or a greater (RSPO3) BMl; and some of them have shown to have a regulatory role in the immune system in patients with RA (WDFY4, BMP7).

\begin{tabular}{llll}
\hline SNP & Gene & $\beta$ Coef. [Cl 95\%] & $p$ \\
\hline rs2746187 & LOC728666/RSPO3 & $2.018[0.949,3.087]$ & $2.646 \times 10^{-4}$ \\
rs8103026 & SIGLEC6/ZNF175 & $-2.135[-3.09,-1.18]$ & $1.724 \times 10^{-5}$ \\
rs2419678 & LOC100132349 & $-2.001[-2.826,-1.175]$ & $6.623 \times 10^{-}$ \\
rs17842463 & SULT2B1 & $-3.515[-5.446,-1.585]$ & $3.402 \times 10^{-3}$ \\
rs1131878 & UGT2B4 & $1.337[0.542,2.131]$ & $1.114 \times 10^{-3}$ \\
rs12757445 & CDC73/KCNT2 & $1.804[0.737,2.871]$ & $1.057 \times 10^{-3}$ \\
rs1638020 & PTPRN2 & $-1.329[-2.1,-0.558]$ & $8.409 \times 10^{-4}$ \\
rs11019575 & MULTIPLE GENES: 399942, 100131364 & $3.356[1.309,4.307]$ & $2.939 \times 10^{4}$ \\
rs72917213 & MEX3C/LOC729051 & $2.319[1.172,3.466]$ & $9.677 \times 10^{-}$ \\
rs6014959 & BMP7 & $-1.942[-3.122,-0.762]$ & $1.434 \times 10^{-}$ \\
rs2870662 & DOK5/CBLN4 & $1.455[0.615,2.295]$ & $7.993 \times 10^{-4}$ \\
rs10776644 & WDFY4 & $-2.159[-3.49,-0.828]$ & $1.661 \times 10^{-3}$ \\
rs7800039 & STEAP4/ZNF804B & $1.564[0.76-2.368]$ & $1.725 \times 10^{-4}$ \\
rs12517451 & ANKRD34B/DHFR & $1.629[0.695,2.562]$ & $7.351 \times 10^{-4}$ \\
rs12722531 & IL2RA & $-3.544[-5.713,-1.376]$ & $1.535 \times 10^{-3}$
\end{tabular}

Conclusions: Our preliminary approach allowed us to select 15 SNPs that may have more relevance related to BMI in patients with early arthritis. However, this is a preliminary study and it is necessary to validate these results in other populations to ensure their involvement in the relationship between the BMI and EA.

Disclosure of Interest: None declared

DOI: 10.1136/annrheumdis-2018-eular.3575

\section{SAT0106 ADVANCES IN THERAPEUTIC MANAGEMENT WITH FIRST BIOLOGICAL THERAPY IN RHEUMATOID ARTHRITIS THROUGHOUT 15 YEARS}

P. Bogas ${ }^{1}$, C. Plasencia ${ }^{1}$, V. Navarro-Compán ${ }^{1}$, D. Benavent ${ }^{1}$, G. Bonilla $^{1}$, L. Nuño ${ }^{1}$, I. Monjo ${ }^{1}$, G. González ${ }^{1}$, J. Molina ${ }^{1}$, A. Balsa ${ }^{1} .{ }^{1}$ Rheumatology, Hospital Universitario La Paz, Madrid, Spain

Background: In the last two decades the treatment in patients with rheumatoid arthritis (RA) has undergone major advances, especially due to the appearance of new therapies, the use of the "treat to target" strategy and a better understanding of the "window of opportunity" concept. However, data from clinical practise confirming the benefits of using these strategies are scarce.

Objectives: To investigate whether the proportion of patients (pts) with RA in maintained remission (R) or low disease activity (LDA) after starting a first biological agent has increased over time and which factors are associated with this change.

Methods: Analysis of a database from a prospective cohort including 365 pts with RA starting a 1st biological agent (BA) (TNF inhibitor, abatacept or tocilizumab) in a tertiary hospital between 2000-2014. Demographic, clinical and analytical data were collected at the beginning of treatment and clinical activity (DAS28) was measured every 6 months. For this study, 3 groups were established according to BA initiation date: interval 1 (i1) (between 2000-2004), (i2) 2005-2009 and (i3) 2010-2014, with a minimum follow-up of 2 years at all pts. For each interval, the percentage of pts achieving maintained (at least 3 consecutive visits) R (DAS28 $<2.6$ ) or LDA (DAS28 <3.2) was determined. In addition, all variables collected were compared between groups by ANOVA and chi square test.

Results: Out of the 365 pts initiating a 1st BA, 133 started in i1, 122 in i2 and 110 in i3. Of these, $38 \%(n=137)$ achieved maintained R/LDA. This percentage increased significantly in successive intervals (31\% in i1 vs, $38 \%$ in i 2 vs $45 \%$ in $i 3, p=0.02)$. Baseline characteristics of pts achieving R/LDA are shown in table $1 \mathrm{~A}$. For patients in i2 and i3, compared to the previous interval (i1 and i2 respectively), a significant higher frequency of use of BA with different mechanisms of action ( $0 \%$ in i 1 vs $2.2 \%$ in i2 vs $34 \%$ in i3, p<0.001), women $(56 \%$ in 11 vs $76 \%$ in i 2 vs $84 \%$ in i $3, \mathrm{p}=0.01)$ and concomitant methotrexate $(56 \%$ in i 1 vs $74 \%$ in i2 vs $81 \%$ in i3, $p=0.03$ ) was found. On the other hand, the percentage of optimized pts increased significantly over time $(13 \%$ in i 1 vs $32 \%$ in i2 vs $56 \%$ in i3, $p<0.001$ table 1B)

\begin{tabular}{|c|c|c|c|c|}
\hline & $\begin{array}{c}2000-2004 \\
(n=41)\end{array}$ & $\begin{array}{c}2005-2009 \\
(n=46)\end{array}$ & $\begin{array}{c}2010-2014 \\
(n=50)\end{array}$ & pvalue \\
\hline \multicolumn{5}{|c|}{ Table 1A: Characteristics at the beginning of biological therapy } \\
\hline Non-TNFi BA (Tocilizumab) & $0 \%$ & $2.2 \%$ & $34 \%$ & $\infty .001$ \\
\hline Age (vears) & $52 \pm 11.1$ & $51 \neq 15.7$ & $54 \pm 121$ & 0.5 \\
\hline Sex (female) & $56 \%$ & $76 \%$ & $24 \%$ & 0.01 \\
\hline Non smokers & $54 \%$ & $61 \%$ & $45 \%$ & 0.6 \\
\hline$B M\left(K g / m^{2}\right)$ & $26.5 \pm 4.7$ & $25.9 \pm 4.4$ & $26 \pm 5,6$ & 0.3 \\
\hline Disesse duration (years) & $9.2 \pm 6.3$ & $10.7 \pm 7.8$ & $7.9 \pm 6.1$ & 0.1 \\
\hline RF * & $85 \%$ & $80 \%$ & $78 \%$ & 0.7 \\
\hline Ant-ccp * & $85 \%$ & $84 \%$ & 85\% & 0.9 \\
\hline DMARDS (MTXJSZPREFL) & $76 \%$ & $89 \%$ & $98 \%$ & 0.02 \\
\hline - Methotrexate & $56 \%$ & $74 \%$ & $81 \%$ & 0.03 \\
\hline - Lefunomide & $7 \%$ & $4 \%$ & $6 \%$ & 0.8 \\
\hline - Salazogyin & $27 \%$ & $35 \%$ & $35 \%$ & 0.7 \\
\hline CPR & $7.3 \pm 10.6$ & $12,1 \pm 12,7$ & $8.7 \pm 11.4$ & 0.001 \\
\hline ESR & $33 \pm 20.5$ & $34 \pm 20.5$ & $19 \pm 10.2$ & $\infty .001$ \\
\hline DAS28 & $5.2 \pm 1.4$ & $4.8 \div 1.1$ & $4.7 \pm 1.1$ & 0.2 \\
\hline HAQ & $8.1 \pm 56$ & $7.9 \pm 5.8$ & $7.9 \times 4.5$ & 0.9 \\
\hline \multicolumn{5}{|l|}{ Table 18: Follow-up characteristics } \\
\hline Follow-up time (years) & $4.0 \pm 2.9$ & $4.7 \pm 2.4$ & $4.6 \pm 2.6$ & 0.06 \\
\hline Time under biologic (years) & $3.8 \pm 2.1$ & $4.4 \pm 2.6$ & $4.1 \pm 2.7$ & 0.2 \\
\hline DAS28 > 3.2 at end of interval (\%) & $23 \%$ & $10 \%$ & $7.5 \%$ & 0.1 \\
\hline Optimized at the end of interval $(\%)$ & $13 \%$ & $32 \%$ & $56 \%$ & $\infty .001$ \\
\hline
\end{tabular}

Conclusions: The percentage of pts with RA achieving maintained R/LDA after initiating a 1st BA has progressively increased over time. This is probably related to a greater use of BAs with different mechanisms of action and concomitant methotrexate. The sustained control of disease activity may allow using more frequently optimized doses of BA.

Disclosure of Interest: None declared

DOI: 10.1136/annrheumdis-2018-eular.4913 